\title{
LOS ESTUDIOS EXITOSOS COMO PRESUPUESTO DEL DERECHO A LOS ALIMENTOS DE LOS HIJOS SOLTEROS MAYORES DE EDAD
}

\author{
SUCCESSFUL STUDIES AS A REQUIREMENT FOR THE RIGHT TO \\ MAINTENANCE OF ADULT SINGLE SONS
}

Sacha Félix Rivas Figueroa* Investigador independiente Lima-Perú https: / / orcid.org/0000-0003-3736-9664 sacharivas@hotmail.com

\section{Resumen}

El presente artículo trata sobre la interpretación de los estudios exitosos de una profesión u oficio previsto en el artículo $424^{\circ}$ del Código Civil como presupuesto del derecho a los alimentos de los hijos solteros (o hijas solteras) mayores de edad. Para ello, se parte de un análisis y comprensión de los conceptos y fundamentos del derecho a los alimentos, la base legal, el Derecho Comparado, la jurisprudencia y todos los presupuestos que configuran el supuesto de hecho regulado con la finalidad de proponer un criterio objetivo como parámetro interpretativo de lo que debemos entender por "éxito en los estudios".

Palabra clave: Derecho de Familia, alimentos, derecho a la educación, estudios exitosos.

\section{Abstract}

This article is about the interpretation of the successful studies of a profession or trade regulated in the article 424 of the Civil Code as a presupposition of the right to maintenance of single sons (or single daughters) with legal age. For this, it starts from an analysis and understanding of the concepts and foundations of the right to maintenance, the law, Comparative Law, jurisprudence and all the presuppositions that set up this case regulated in order to propose an objective criterion as an interpretive parameter of what we should understand by "success in studies".

Keyboards: Family Law, maintenance, right to education, successful studies.

Abogado por la UNMSM. Magíster en Derecho Civil por la PUCP. Juez Especializado en Familia de la Corte Superior de Justicia de Lima. 


\section{INTRODUCCIÓN}

El artículo $424^{\circ}$ del Código Civil (en adelante: CC) modificado por la Ley $\mathrm{N}^{\circ} 27646$ prescribe que los padres tienen la obligación de proveer al sostenimiento de los hijos e hijas, solteros y mayores de dieciocho años, que estén siguiendo con éxito estudios de una profesión u oficio hasta que tengan 28 años de edad, es decir, la ley obliga a los padres a seguir brindando alimentos a sus hijos que adquirieron la mayoría de edad, siempre y cuando, se presenten dichos presupuestos de forma copulativa.

Más allá de la discusión si, jurídicamente, es una obligación o un deber o "derecho-deber" de los padres (Díez-Picazo y Gullón, 1998), brindar alimentos a sus hijos; en la práctica, vemos que la cuestión suele centrarse en lo que debemos entender por: "éxito en los estudios" o simplemente: "éxito". Particularmente, este último término ha derivado en profusas discusiones, no solo de abogados, jueces, fiscales, estudiantes y personas vinculadas al Derecho; sino también, de los propios litigantes y público en general, cuando han tenido la oportunidad de alegar, defender, decidir, o simplemente, opinar sobre el tema.

Recuerdo una publicación de una revista jurídica digital en la red social Facebook, que hacía mención a la sentencia de un juez de paz letrado, mediante la cual otorgaba una pensión de alimentos a una hija soltera mayor de edad que cursaba estudios universitarios y tenía como nota un promedio ponderado de 11.71. Los abogados y demás internautas se enfrascaron en interminables debates opinando si era "justo" o no que su padre le deba dar alimentos. Se apreciaba, además, un evidente sesgo en las posturas asumidas, pues, "curiosamente", la mayoría de hombres jóvenes y las mujeres apoyaban la decisión; mientras que los hombres adultos de mediana edad solían rechazarla.

Como juez de familia, desde que reviso en segundo grado las apelaciones de las sentencias de los juzgados de paz letrado, mi posición siempre ha sido considerar como exitosos los estudios de un alumno con un promedio ponderado igual o mayor de 11, siempre y cuando, tenga la posibilidad académica de proseguir y culminar sus estudios; y más adelante explicaré por qué asumo tal postura. Así lo he plasmado en las sentencias de vista que he emitido. No obstante, soy consciente que las críticas continuarán, ya que más allá de los argumentos que se expongan, hay intereses (como una pensión de alimentos) en juego. 
De todos modos, debe quedar claro que no pretendo acabar con la discusión, sino brindar algunos aportes teóricos y prácticos que nos den más luces al momento de abordar el tema.

Los métodos que he utilizado son el exegético, dogmático, el Derecho Comparado y la jurisprudencia, habiendo tenido dificultades para acceder a una mayor cantidad de fuentes bibliográficas y a las fuentes históricas como lo hubiera querido- debido a que las bibliotecas estuvieron cerradas por la pandemia; sin embargo, espero que estas deficiencias no hayan logrado afectar significativamente el producto final, el cual pongo a su consideración.

\section{LOS ALIMENTOS:}

No podemos abordar el tema de los estudios exitosos sin antes tratar sobre la institución de los alimentos.

Con mucho acierto, Barbero (citado por Díez-Picazo y Gullón, 1998) ha indicado que "el primer bien que una persona posee en el orden jurídico es su vida, el primer interés que tiene es su conservación y la primera necesidad con la que se enfrenta es procurarse los medios para ello" (p. 47).

Ya en la primera mitad del siglo pasado, Josserand (citado por Varsi, 2012) definía a la "obligación alimentaria" como "el deber impuesto jurídicamente a una persona de asegurar su subsistencia de la otra; (...)" (p. 420).

Cabanellas (1979), en su reconocido diccionario, sostenía que los alimentos son "asistencias que en especie o en dinero, y por ley, contrato o testamento, se dan a una o más personas para su manutención y subsistencia; esto es, para comida, bebida, vestido e instrucción cuando el alimentista es menor de edad" (p. 252).

En nuestro medio, Cornejo (1999), escribía lo siguiente sobre los alimentos:

Comprenden todo lo necesario para el sustento, habitación, vestido y asistencia médica del alimentista, según su rango y condición social (alimentos congruos). Excepcionalmente, pueden restringirse a lo estrictamente requerido para la subsistencia (alimentos necesarios); o, a la inversa, extenderse a lo que demandan la educación einstrucción profesional del alimentista (como ocurre cuando se trata de menores). (p. 568) 
Hasta aquí, vemos los alimentos son los medios para mantener la subsistencia del alimentista, ya sea de manera directa (sustento, habitación, vestido, etc.) o de manera indirecta (educación, capacitación para el trabajo); sin embargo, está concepción ha ido evolucionando, y juristas como Aguilar Cornelio (citado por Varsi, 2012), a mediados de la década de los noventas, sostenía que la "obligación alimentaria" respecto de los hijos "no termina tan solo con la provisión de elementos materiales necesarios para su supervivencia, sino que, se hace extensivo a su formación integral; hasta que estén debidamente capacitados para subvenir decorosamente a su propia subsistencia" (p. 420).

De este modo, la noción de los alimentos ya no se reduce a la cobertura de las necesidades fisiológicas del alimentista, sino también, y con la misma importancia, a sus necesidades mentales y emocionales, es decir, implica todo lo necesario para su subsistencia digna o decorosa ${ }^{41}$.

En la actualidad, luego de haber sido modificado el año 2014 mediante la Ley $\mathrm{N}^{\circ} 30292$, el artículo $472^{\circ}$ del CC explica lo siguiente sobre esta institución: "Se entiende por alimentos lo que es indispensable para el sustento, habitación, vestido, educación, instrucción y capacitación para el trabajo, asistencia médica y psicológica y recreación, según la situación y posibilidades de la familia. (...)".

Como se observa, si bien los alimentos siguen estando sujetos a los presupuestos de necesidad del alimentista y la posibilidad del alimentante (art. $481^{\circ}$ del CC), se ha ampliado su contenido hasta incorporar aspectos como la capacitación para el trabajo, la asistencia psicológica y la recreación ${ }^{42}$, los cuales, entendemos, operan para todos los alimentistas sin importar su edad, pues el artículo $2^{\circ}$ de la Ley $\mathrm{N}^{\circ} 30292$ también derogó el segundo párrafo del artículo $472^{\circ}$ del CC que señalaba: "Cuando el alimentista es menor de edad, los alimentos comprenden también su educación, instrucción y capacitación para el trabajo".

Aquello es comprensible, si en la vida diaria o por nuestro trabajo, conocemos casos en los que un joven estudiante requiere asistencia psicológica por estar padeciendo de estrés agudo o una persona adulta

${ }_{41}$ En ese mismo sentido, Chunga (2011), afirma que los “alimentos implica no solamente la palabra propiamente dicha, sino que abarca más allá del significado; en el sentido más extenso, es todo lo que nos ayuda a protegernos para poder vivir y desarrollarnos de forma digna" (p. 160). Similar postura asume el profesor Varsi (2012) cuando explica que "El concepto de alimentos apunta a la satisfacción de las necesidades básicas del ser humano que se dan, tanto en el aspecto material, entiéndase comida, vestido, alimentos propiamente dichos, como en el aspecto espiritual o existencial (...)" (p. 419).

42 Desde el año 1992, la recreación estaba incluida en los alimentos de los menores de edad en virtud del extinto Código de los Niños y Adolescentes (CNA) (Art. 101 del Decreto Ley 26102). 
mayor necesita recrearse para mantenerse sana y activa, por solo poner dos ejemplos.

Respecto de los hijos, los alimentos son un derecho fundamental plasmado en el artículo $6^{\circ}$ de la Constitución Política del Perú de la siguiente manera: "es deber y derecho de los padres alimentar, educar y dar seguridad a sus hijos".

\section{FUNDAMENTO Y PRESUPUESTOS DEL DERECHO A LOS ALIMENTOS:}

Señala Jiménez (2012), que si bien algunos autores encuentran el fundamento del derecho a los alimentos en el derecho a la vida del alimentista; en el vínculo parental o en otras razones; la doctrina mayoritaria admite que su fundamento es el principio de solidaridad familiar (pp. 89 y 90$)^{43}$.

Por otro lado, es pacífico en la doctrina nacional (Cornejo, 1999; Hernández, 2011; Varsi, 2012; Aguilar, 2013), que los presupuestos o condiciones para que nazca el derecho a los alimentos son los siguientes:

1. El estado de necesidad del alimentista.

2. Las posibilidades del alimentante; $y$

3. La norma legal que establezca la obligación.

\subsection{El estado de necesidad del alimentista}

Consiste en la imposibilidad de proveerse los alimentos por sí mismo, bien sea por razones intrínsecas al individuo (inmadurez, enfermedad, discapacidad, ancianidad, etc.) o por razones extrínsecas a este (desempleo, huelga, etc.). Para Bossert y Zanonni (2016) "se traduce en un estado de indigencia o insolvencia que impide la satisfacción de los requerimientos alimentarios ... lo fundamental es que se carezca de medios económicos que permitan sufragar las necesidades" (p. 41).

Por su parte, Díaz-Picazo y Gullón (1998), nos explican lo siguiente:

43 Vid. Díez-Picazo y Gullón (1998), Bossert y Zannoni (2016) y Varsi (2012). 
A partir del momento en que se adquiere determinada capacitación, la lucha por la vida es asunto personal que cada uno ha de resolver, pero no se puede dejar de tomar en consideración el fracaso en esa lucha (v gr., el paro) o la imposibilidad de tomar parte en ella (v gr., niños, enfermos, disminuidos físicos y psíquicos, personas de la llamada tercer edad). (p. 47)

De manera similar, Cornejo (1999), ha sostenido:

Dotado el hombre por la naturaleza de las aptitudes y virtualidades más relevantes (sic) entre todos los seres animales está, sin embargo, sujeto, como lo están todos los animales inferiores y aun con mayor intensidad que muchos de ellos, a un fenómeno inevitable: el de pasar, durante la primera etapa de su vida, por una situación de insuficiencia que lo inhabilita para valerse por sí mismo. ... Parecida situación se produce cuando, salido ya el ser humano de aquella primera etapa de su vida, cae, sin embargo, en insuficiencia o desamparo por causa de otros factores -enfermedad, accidente, vejez, desempleo $u$ otros semejantes - menos generales pero igualmente graves al menos para la supervivencia del ser individual. Varían, pues, los hechos que originan el desamparo - inmadurez, accidente, enfermedad, vejez, desocupación-; ... (p. 567)

Como se aprecia, el estado de necesidad es, a fin de cuentas, una situación de hecho desventajosa, y los motivos que originan dicha situación son variados. Lo que queremos resaltar, a los fines de este artículo, es que la doctrina admite que factores como la inmadurez y el desempleo pueden desencadenar un estado de necesidad.

La jurisprudencia nacional ha dejado establecido, que, en el caso de los menores de edad, su estado de necesidad se presume ${ }^{44}$.

\subsection{Las posibilidades del alimentante}

Es evidente que solo puede exigírsele prestar alimentos a quien se encuentra en condiciones de atender sus propias necesidades, ya que lo contrario, sería poner en riesgo su propia subsistencia. Efectivamente, si el obligado a brindar alimentos también se encuentra en estado de necesidad, entonces, ambos serían los acreedores alimentarios de quienes, por ley, se encuentran en el orden de prelación más próximo (arts. $475^{\circ}$ y $476^{\circ}$ del CC).

44 Casación N 3874-2007-TACNA de fecha 13 de octubre de 2008 emitida por la Sala Civil Transitoria de la Corte Suprema de Justicia de la República. 
Varsi (2012) aclara que "en este caso predomina el derecho a conservar la propia existencia" (p. 422) ${ }^{45}$.

Para determinar si el obligado se encuentra en posibilidades de cubrir los alimentos del requirente, nos dice Jiménez (2012), "se atenderá a su activo como a su pasivo".

Considerando que los alimentos pueden prestarse en especie y en dinero, dentro del activo, además de los bienes con los que cuenta el obligado, debemos tener en cuenta sus ingresos periódicos. Sobre esto último, Varsi (2012) nos explica lo siguiente:

Los ingresos de una persona se pueden definir como todos aquellos montos de dinero o especies que "ingresan" a la esfera de dominio de esta y que pueden ser utilizados a total discreción de la persona. Es una gran categoría que incluye el total de ingresos tales como sueldos, bonificaciones especiales, horas extras, aumentos, vacaciones, escolaridad, dietas, aguinaldos, asignaciones especiales, donaciones, utilidades, alquileres, ganancias, intereses y demás ingresos adicionales, etc.". (p. 474)

Como vemos, para que sean considerados ingresos deben ser de libre disposición. De esta manera, las sumas de dinero brindadas por el empleador como condiciones de trabajo (viáticos, movilidad, etc.) no serían ingresos, ya que son entregados con la finalidad de que el trabajador pueda prestar sus servicios, debiendo dar cuenta al empleador sobre el destino de dicho dinero.

Respecto, del pasivo, Jiménez (2012) nos informa que se debe considerar las deudas del obligado y "especialmente sus necesidades y las de su familia, a las que debe atender en primer término" (p. 101). En este último caso, se refiere a los alimentos que el deudor alimentario debe prestar a aquellos que se encuentran en igual o en un primer orden de prelación.

Una vez evaluado el activo y el pasivo del obligado, recién se puede establecer si se encuentra en posibilidades de prestar los alimentos.

45 Recuerdo un caso de dos personas adultas mayores de edad avanzada (más de setenta años) que se encontraban separadas de hecho. La cónyuge demandó los alimentos al cónyuge, pero este último solo vivía de una exigua pensión y de lo que le daba, voluntariamente, uno de sus hijos, es decir, ambos se encontraban en estado de necesidad. Ante esta situación, lo correcto hubiese sido que la cónyuge demande los alimentos a sus hijos en mérito al artículo $478^{\circ}$ del CC. 


\subsection{La norma legal que establezca la obligación}

Cornejo (1999) nos dice que como es una obligación civil y no una obligación natural, debe estar plasmada en la ley.

Sin perjuicio de que el derecho a los alimentos de los hijos es de origen constitucional, en general, el derecho a los alimentos surge del vínculo conyugal y de parentesco previsto en la ley. Es así, que los arts. $474^{\circ}$ y $475^{\circ}$ del CC concordado con el art. $93^{\circ}$ del CNA (en el caso de los menores de edad), determinan quienes son los obligados a prestar alimentos y su orden de prelación.

La excepción a la regla precedente la constituye el art. $766^{\circ}$ del CC, ya que prevé el legado de alimentos, lo que significa que el beneficiario, en este caso, puede ser o no ser pariente del testador.

Por el contrario, otras prestaciones económicas con fines asistenciales no pueden considerarse alimentos si la ley así no lo establece (p. ej., la renta vitalicia).

\section{ANTECEDENTES Y REGULACIÓN LEGAL DE LOS ESTUDIOS EXITOSOS:}

El derecho a los alimentos de los hijos que están siguiendo con éxito estudios de una profesión u oficio, estaba regulado en el CC de 1936 de la siguiente manera:

Artículo 399.- Subsiste la obligación impuesta en el inciso 1 del artículo anterior respecto de los hijos que estén siguiendo con éxito una carrera $\mathrm{u}$ oficio, y de las hijas solteras que no se encontraren en estado de ganarse la vida.

En este caso, dada la antigüedad de la disposición, aún había una discriminación hacia las hijas, pues para ellas se aplicaba otro presupuesto asumiendo que no eran capaces o que no debían estudiar una carrera $u$ oficio; también se asumía que eran las únicas que, a pesar de su mayoría de edad, podían quedar en desamparo. Tampoco se establecía una edad límite para la formación laboral. Aunque no se hacía referencia a los estudios, se entiende que estaba implícitamente comprendido, pues si interpretamos que la carrera $u$ oficio del hijo es exitosa, no habría razón para darle alimentos. 
Con la promulgación del CC de 1984, hubo una pequeña modificación; sin embargo, continuaba la discriminación hacia las hijas. El artículo quedó redactado de esta forma:

Artículo 424.- Subsiste la obligación de proveer al sostenimiento de los hijos e hijas mayores de dieciocho años que estén siguiendo con éxito una profesión u oficio, y de las hijas solteras que no se encuentren en aptitud de atender a su subsistencia.

En el año 2002, mediante la Ley 27646, se modificaron los artículos $424^{\circ}$, $473^{\circ}$ y $483^{\circ}$ del CC, y finalmente, se introdujeron los demás presupuestos que, en la actualidad, configuran el derecho a los alimentos de los hijos mayores de edad. Así, el artículo $424^{\circ}$ del CC establece lo siguiente:

Subsiste la obligación de proveer al sostenimiento de los hijos e hijas solteros mayores de dieciocho años que estén siguiendo con éxito estudios de una profesión u oficio hasta los 28 años de edad; y de los hijos e hijas solteros que no se encuentren en aptitud de atender a su subsistencia por causas de incapacidad física o mental debidamente comprobadas.

Más allá de que la disposición normativa se encuentra, erróneamente, ubicada en el Título III de la Sección Tercera del Libro III del Código Civil, referente a la institución de la patria potestad -como bien lo anotó el profesor Varsi (2012)-, guarda coherencia con el artículo $483^{\circ}$ del CC, el cual establece lo siguiente:

El obligado a prestar alimentos puede pedir que se le exonere si disminuyen sus ingresos, de modo que no pueda atenderla sin poner en peligro su propia subsistencia, o si ha desaparecido en el alimentista el estado de necesidad. Tratándose de hijos menores, a quienes el padre o la madre estuviesen pasando una pensión alimenticia por resolución judicial, esta deja de regir al llegar aquéllos a la mayoría de edad. Sin embargo, si subsiste el estado de necesidad por causas de incapacidad física o mental debidamente comprobadas o el alimentista está siguiendo una profesión u oficio exitosamente, puede pedir que la obligación continúe vigente.

Si concordamos ambas disposiciones, concluimos que el alimentista al que se hace referencia en la última parte del artículo $483^{\circ}$ es el hijo soltero (o soltera) del art. 424 del CC, quien debe estar siguiendo con éxito estudios de una profesión u oficio hasta una edad límite de 28 años, para que mantenga el derecho a recibir alimentos de sus padres. 
Extrañamente, entre ambos artículos, se encuentra el artículo $473^{\circ}$ del CC, que también fue modificado por la Ley № 27646 con este tenor:

El mayor de dieciocho años solo tiene derecho a alimentos cuando no se encuentre en aptitud de atender a su subsistencia por causas de incapacidad física o mental debidamente comprobadas.

Si la causa que lo redujo a ese estado fue su propia inmoralidad, solo podrá exigir lo estrictamente necesario para subsistir.

No se aplica lo dispuesto en el párrafo anterior, cuando el alimentista es ascendiente del obligado a prestar alimentos.

Ahora bien ¿Cómo concordamos este artículo con los anteriores si es aparentemente contradictorio? Pues, nuevamente, recurrimos a la interpretación sistemática del CC para solucionar la deficiente técnica legislativa. Si ya hemos dicho y resulta claro que los hijos solteros que estén siguiendo con éxito estudios de una profesión u oficio hasta un límite de 28 años de edad, tienen derecho a recibir alimentos de sus padres, entonces, se entiende que la primera disposición del artículo $473^{\circ}$ del CC está referida a aquellos mayores de edad que no se encuentran en dicho supuesto ${ }^{46}$. Por ejemplo: Los ascendientes, los hermanos, los hijos mayores de edad casados (solo les correspondería alimentos si no pudiesen atender a su subsistencia por causas de incapacidad física o mental debidamente comprobadas, siendo en este caso, los obligados de primer orden, sus cónyuges [art. $475^{\circ}$ del CC]), etc.

De lo expuesto, advertimos que son 2 los supuestos de hecho en los que les corresponde, a los hijos (e hijas) que han adquirido la mayoría de edad, seguir recibiendo alimentos de sus padres:

1. Cuando sean solteros (o solteras) y estén siguiendo con éxito estudios de una profesión $u$ oficio hasta la edad de 28 años.

2. Cuando sean solteros (o solteras) y no se encuentren en aptitud de atender a su subsistencia por causas de incapacidad física o mental debidamente comprobadas.

46 En principio, conforme al artículo $473^{\circ}$ del CC, al hijo o hija mayor de edad que no se encuentre en aptitud de atender a su subsistencia por causas de incapacidad física o mental debidamente comprobadas, le corresponde recibir alimentos; sin embargo, si aquel hijo o hija aún no contrajo nupcias, por el principio de especialidad, se le debe aplicar el artículo $424^{\circ}$ del CC, ya que serán sus padres los obligados de primer orden. 
Respecto del primer supuesto, siendo el objeto de estudio de este artículo, lo desarrollaremos más adelante.

Con relación al segundo supuesto, es evidente que los hijos solteros mantendrían su derecho a recibir alimentos de sus padres, pues se encuentran en estado de necesidad, que es uno de los presupuestos o condiciones del derecho a los alimentos; sin embargo, considerando que ya han adquirido la mayoría de edad, la exigencia de la probanza de su condición incapacitante (para el trabajo) es mayor ${ }^{47}$.

Como habrán notado, este supuesto también está recogido, de manera general, en el primer párrafo del artículo $473^{\circ}$ del CC.

\section{EL DERECHO COMPARADO:}

En España, los hijos solteros mayores de edad (18 años) tienen derecho a recibir alimentos de sus padres, siempre y cuando, se encuentren en estado de necesidad, es decir, es irrelevante si están siguiendo con éxito estudios de una profesión u oficio o no, lo importante es que no puedan atender a su propia subsistencia con los medios y recursos con los que cuentan.

Ello se desprende del artículo $152^{\circ}$ del CC español que dice:

Cesará también la obligación de dar alimentos:

3. ${ }^{\circ}$ Cuando el alimentista pueda ejercer un oficio, profesión o industria, o haya adquirido un destino o mejorado de fortuna, de suerte que no le sea necesaria la pensión alimenticia para su subsistencia.

Cabe acotar que en España existe la figura de la emancipación, por la cual, el mayor de 16 años y menor de 18, está facultado para regir sobre su persona y bienes como si fuese mayor de edad, con solo algunas

47 Debe diferenciarse la discapacidad sensorial, motora, mental o psicosocial de la incapacidad física o mental para el trabajo. En la actualidad, la discapacidad es entendida como una discapacidad social, es decir, los que "discapacitan" a las personas con deficiencias físicas y mentales son la sociedad y el Estado. La mayoría de personas con discapacidad pueden integrarse al trabajo si se les brinda los medios y las condiciones adecuadas, por lo que solo un pequeño sector de aquellos estará, efectivamente, incapacitado física y mentalmente para el trabajo (p. ej., Las personas con cuadriplejia). 
restricciones (arts. $314^{\circ}, 317^{\circ}, 320^{\circ}$ y $323^{\circ}$ del CC español), por lo que Jiménez (2012) considera que "la mera mayoría de edad no conllevará la automática pérdida del derecho a alimentos, sino que este se producirá con la emancipación, en el sentido de vida independiente, siempre que el hijo no incurra en situación de necesidad" (p. 92).

De este lado del mundo, países como Argentina, Colombia y Chile han establecido disposiciones legislativas para presumir el estado de necesidad del hijo mayor de edad (mayor de 18 años) y menor de 21 años. Así, el artículo $658^{\circ}$ del Código Civil y Comercial de la Nación (En adelante: "CCC") argentino señala lo siguiente:

Regla general. Ambos progenitores tienen la obligación y el derecho de criar a sus hijos, alimentarlos y educarlos conforme a su condición y fortuna, aunque el cuidado personal esté a cargo de uno de ellos.

La obligación de prestar alimentos a los hijos se extiende hasta los veintiún años, excepto que el obligado acredite que el hijo mayor de edad cuenta con recursos suficientes para proveérselos por sí mismo.

En el caso de Colombia, con un evidente sesgo discriminatorio, el artículo $422^{\circ}$ del CC colombiano textualmente indica:

Los alimentos que se deben por ley, se entienden concedidos para toda la vida del alimentario, continuando las circunstancias que legitimaron la demanda.

Con todo, ningún varón de aquéllos a quienes solo se deben alimentos necesarios, podrá pedirlos después que haya cumplido veintiún años, salvo que por algún impedimento corporal o mental, se halle inhabilitado para subsistir de su trabajo; pero si posteriormente se inhabilitare, revivirá la obligación de alimentarle ${ }^{48}$.

Sobre esta política familiar, de presumir el estado de necesidad de los hijos menores de 21 años de edad y el correspondiente derecho a los alimentos de sus padres, Bossert y Zannoni (2016) nos dan algunas luces al respecto:

48 Debemos aclarar, que el CC colombiano aún mantiene la distinción entre hijos legítimos e ilegítimos $\left(\operatorname{art.} 411^{\circ}\right)$, habiéndose dispuesto en el art. $414^{\circ}$ que a los hijos legítimos le corresponden los alimentos congruos (los que le permiten subsistir modestamente de acuerdo a su posición social) y a los hijos ilegítimos, los alimentos necesarios (los que "bastan para sustentar la vida"). De esta manera, habría un régimen distinto para los hijos mayores de edad, dependiendo de su sexo y su origen, lo que no se condice con el derecho fundamental a la igualdad. El caso ecuatoriano es idéntico al colombiano, con la sola diferencia que reduce la edad de presunción del estado de necesidad de los hijos ilegítimos a la minoría de edad (art. $360^{\circ}$ del CC ecuatoriano). 
Fijar la mayoría de edad a los dieciocho años responde a una tendencia universal. Sin embargo, entre nosotros, se advierte en el legislador algo así como un paternalismo a priori que se apoya más en la convicción de que subsiste en esos jóvenes una inmadurez, falta de criterio o vulnerabilidad, que debe ser suplida por el padre o madre con quien conviven. (p. 394)

No obstante ello, debemos precisar que Argentina y Chile, al igual que Bolivia, cuentan con disposiciones similares a la nuestra para beneficiar con alimentos a los hijos mayores de edad que cursan estudios de una profesión $\mathrm{u}$ oficio.

Así tenemos que el artículo $663^{\circ}$ del CC argentino establece lo siguiente:

Hijo mayor de edad que se capacita. La obligación de los progenitores de proveer recursos al hijo subsiste hasta que este alcance la edad de veinticinco años, si la prosecución de estudios o preparación profesional de un arte $\mathrm{u}$ oficio, le impide proveerse de medios necesarios para sostenerse independientemente.

Pueden ser solicitados por el hijo o por el progenitor con el cual convive; debe acreditarse la viabilidad del pedido.

En este caso, no se hace alusión al estado civil del hijo o hija (si es soltero o casado); tampoco al éxito de los estudios (o preparación) y se fijó la edad límite del alimentista para que lleve dichos estudios, en 25 años. Por la forma como está redactado el artículo, se desprende que los estudios por sí solos, no son suficientes para acceder al derecho a los alimentos, sino que dichos estudios deben de ser la causa del estado de necesidad del hijo.

Como ya dijimos, el CC chileno, si bien contempla la presunción del estado de necesidad para el hijo mayor de edad y menor de 21 años, también beneficia con alimentos al hijo que esté estudiando una profesión u oficio hasta que cumpla los 28 años de edad. El art. $332^{\circ}$ del CC de dicho país dispone lo siguiente:

Los alimentos que se deben por ley se entienden concedidos para toda la vida del alimentario, continuando las circunstancias que legitimaron la demanda.

Con todo, los alimentos concedidos a los descendientes y a los hermanos se devengarán hasta que cumplan veintiún años, salvo que estén 
estudiando una profesión u oficio, caso en el cual cesarán a los veintiocho años; que les afecte una incapacidad física o mental que les impida subsistir por sí mismos, o que, por circunstancias calificadas, el juez los considere indispensables para su subsistencia.

Como observamos, el CC chileno tampoco establece como un presupuesto del derecho a los alimentos de estos hijos que sus estudios sean exitosos; no obstante ello, teniendo en cuenta que el art. $332^{\circ}$ del CC chileno fue modificado por la Ley 19.595 publicada con fecha 26 de octubre de 1998, y por lo tanto, es anterior a nuestra Ley $27646^{49}$, que modificó los artículos $424^{\circ}, 473^{\circ}$ y $483^{\circ}$ del CC, es probable que haya sido el CC chileno el inspirador de nuestros legisladores al momento de incrementar a 28 años el límite de edad del alimentista que se capacita para el trabajo.

Distinto es el caso de la legislación boliviana, la cual, en el artículo $109^{\circ}$ del Código de las Familias y del Proceso Familiar de 2014 (En adelante: "CFPF"), ha previsto lo siguiente sobre la asistencia familiar ${ }^{50}$ :

II. La asistencia familiar se otorga hasta cumplida la mayoría de edad, y podrá extenderse hasta que la o el beneficiario cumpla los veinticinco (25) años, a fin de procurar su formación técnica o profesional o el aprendizaje de un arte $\mathrm{u}$ oficio, siempre y cuando la dedicación a su formación evidencie resultados efectivos.

Es así que, de lo que hemos investigado, Bolivia es el único país de la región que contiene una norma parecida a la peruana sobre los estudios exitosos, no pudiendo dejar de apreciar, además, que el énfasis de aquellos estudios formativos está en los "resultados efectivos" obtenidos.

\section{EL PRESUPUESTO DE LOS ESTUDIOS EXITOSOS:}

En el punto II del presente artículo, ya hemos hecho mención a los presupuestos del derecho a los alimentos; sin embargo, debemos aclarar que estos son los presupuestos "generales", que, en toda circunstancia, deben concurrir para acceder a aquel derecho. Sin embargo, hay casos particulares en los que se requiere algún presupuesto adicional y específico. Uno de ellos es el regulado en la primera parte del art. $424^{\circ}$ del CC.

Como ya hemos dicho, el artículo $424^{\circ}$ del CC prescribe que: "Subsiste la obligación de proveer al sostenimiento de los hijos e hijas solteros mayores

Promulgada el 21 de enero de 2001 y publicada el 23 de enero de 2002.

Nomen iuris equivalente a "alimentos". 
de dieciocho años que estén siguiendo con éxito estudios de una profesión u oficio hasta los 28 años de edad; ...

A continuación, vamos a analizar dicho artículo para obtener los presupuestos, que llamaremos "específicos", del derecho a los alimentos en tal supuesto de hecho.

Los presupuestos "específicos" del derecho a los alimentos en el artículo en mención serían:

\section{a. El vínculo filial:}

Como lo establece la norma, los beneficiarios de los alimentos solo pueden ser los hijos (o hijas); y ello tiene su origen en el "derecho-deber" que impone la patria potestad a los padres, de proveer al sostenimiento y la educación de los hijos menores de edad (art. $74^{\circ}$ inc. b) del CNA). Si nos guiamos por el inciso b) del art. $77^{\circ}$ del CNA, la patria potestad se extingue al adquirir el hijo la mayoría de edad, lo que conllevaría a que cese automáticamente la obligación alimentaria del padre; sin embargo, considerando aspectos como la inmadurez del hijo o el desempleo que afecta a muchos jóvenes en el país y los coloca en un estado de necesidad, el legislador ha previsto prolongar aquel "derecho-deber" de los padres en beneficio de los hijos.

\section{b. El estado civil de soltero o soltera:}

Como ya hemos visto, ninguna legislación extranjera hace un distingo en el estado civil de los hijos para que opere la obligación alimentaria de los padres, y considero que es lo más razonable, ya que, en virtud del art. $474^{\circ}$ del CC, padres e hijos se deben alimentos recíprocamente, siempre y cuando, concurran los presupuestos "generales".

Parece ser, que el legislador nacional ha estimado que si el hijo o hija mayor de edad ha contraído nupcias, conforme al art. $475^{\circ}$ del CC, debería ser su cónyuge (y no sus padres) quien le brinde los alimentos en caso los requiera; sin embargo, la prelación que establece el art. $475^{\circ}$ del CC no excluye a los otros obligados de prestar los alimentos, en caso no hubiera un obligado precedente o este último no se encuentre en posición de poder hacerlo.

c. El rango de edad entre los 18 y los 28 años: 
Se desconocen las razones por las cuales el legislador nacional ha fijado en 28 años el límite de edad de los hijos para tener derecho a los alimentos por motivo de estudios exitosos, ya que no se ha podido acceder al proyecto de la Ley $27646^{51}$. No obstante, hemos hecho notar, que unos años antes, el CC chileno ya preveía dicha edad como la máxima para acceder a los alimentos en caso los hijos estén estudiando, por lo que pudiera ser que nuestro legislador se inspiró en el ordenamiento jurídico vecino.

Asumiendo que nuestros legisladores conocían el sistema educativo peruano, podemos inferir los motivos por los que estableció en 28 años el tope de edad de los hijos que estudian para que puedan acceder a los alimentos.

Según el art. $36^{\circ}$ de la Ley 28044 - Ley General de Educación (En adelante: LGE), la educación inicial se brinda de forma escolarizada hasta los 5 años de edad, por lo que un niño debería iniciar la educación primaria a partir de los 6 años de edad. Si el estudiante culmina los seis años de educación primaria y los cinco años de educación secundaria sin desaprobar ningún año, entonces, culminaría toda su educación básica a los 16 o 17 años de edad, en promedio.

Ahora bien, nuestro sistema educativo carece de una etapa intermedia o "puente" entre la educación básica y la educación superior, que en otros países suele llamarse "bachillerato" 52 o "escuela preparatoria", por lo que, salvo excepciones, nuestros jóvenes recién egresados del colegio, suelen capacitarse en academias o centros preuniversitarios antes de postular a una vacante en una institución de educación superior (universidad, instituto o escuela de educación superior). Todo ello, hace que muchos de nuestros jóvenes no alcancen una vacante en la primera oportunidad que postulan.

Por otro lado, dependiendo de la institución educativa de la que egresa el postulante, la carrera que elija y la institución a la que postula, se le hará, más o menos difícil, ser admitido como alumno. Por ejemplo: No es lo mismo postular a la carrera de Medicina Humana que a una carrera técnica.

Y hablando de Medicina Humana, es la carrera profesional más larga del Perú. El pregrado dura 7 años (14 semestres), por lo que si tomamos en

51 Proyecto de ley $\mathrm{N}^{\circ} 00503$ presentado con fecha 18 de setiembre de 2001 por la ex congresista Judith de la Matta. Si bien no se ha podido acceder al proyecto, se ha revisado el dictamen de la Comisión de Justicia del Congreso sobre aquello; sin embargo, no se hace ninguna referencia a los motivos por los cuales se establecía como límite de edad los 28 años.

52 En el Perú se denomina "bachillerato" al primer grado académico obtenido una vez culminada la educación superior. 
cuenta que la obtención del título profesional que habilite, al graduado de esta carrera, a ejercer su profesión, puede durar otro año más, entonces, la carrera completa podría durar, mínimamente, unos 8 años.

Estos podrían ser algunos de los motivos por los que el legislador ha estimado en 28 años el límite de edad de los hijos para que les corresponda recibir alimentos de sus padres por proseguir estudios exitosos.

\section{d. Los estudios exitosos de una profesión u oficio:}

La jurisprudencia ha establecido que los estudios a los que se refieren este presupuesto, también comprenden los estudios preparatorios o previos, requeridos para el acceso a los estudios superiores. Así, en la Casación $\mathrm{N}^{\circ}$ 3016-2002-LORETO de fecha 21 de febrero de 2003, la Sala Civil Transitoria de la Corte Suprema ha señalado:

Sexto.- Que, si bien para poder acceder a los estudios superiores, se tiene que pasar por las etapas o estudios pre profesionales, como son los estudios primarios, secundarios o preuniversitarios, es decir academias de ingresos a universidades, el artículo cuatrocientos ochentitrés (sic) del Código Civil, en todo caso está referido a cursar estudios exitosamente.

De manera similar, en la Casación № 1338-2004-LORETO de fecha 13 de setiembre de 2005, la Sala Civil Transitoria de la Corte Suprema ha remarcado:

Quinto.- Que, conforme ha establecido jurisprudencialmente esta Sala Civil Transitoria, si bien es cierto que el último párrafo del artículo cuatrocientos ochentitrés (sic) del Código Civil, únicamente se refiere a "seguir" una profesión $\mathrm{u}$ oficio, y no alude al verbo "estudiar", debe entenderse que la norma abarca igualmente a los estudios tendientes a obtener una profesión $u$ oficio, que incluye a los estudios preparatorios -primarios, secundarios o para el ingreso a estudios superiores- y que solo en estos casos puede permitirse que un hijo mayor de edad pueda seguir percibiendo alimentos, siempre que curse dichos estudios de manera exitosa ...

Muestro mi conformidad con el criterio asumido por la Corte Suprema al interpretar de manera extensiva el art. $483^{\circ}$ del CC, de tal manera que los estudios de una profesión u oficio implican los estudios previos requeridos para poder iniciarlos. Debemos de tener en cuenta, además, que la LGE, 
no solo contempla a la educación básica regular, sino también, a otras modalidades educativas como la educación técnico-productiva, por lo que podría darse el caso que el hijo se esté capacitando para el trabajo mientras está cursando el nivel de educación secundaria.

Ahora bien, como hemos visto en el punto IV, países como Argentina y Chile, que cuentan con una norma similar a la nuestra, no exigen como condición que los estudios de los hijos sean exitosos para que proceda el derecho a los alimentos ${ }^{53}$. Entonces ¿Cuál sería la razón para que nuestro código sí establezca este requisito adicional? Creemos que se desprende del art. II del Título Preliminar del CC, esto es, de la prohibición del ejercicio abusivo del derecho.

Efectivamente, no estamos a favor de casos, en los que el hijo mayor de edad se pone a estudiar diversas carreras sin terminarlas con la finalidad de que sus padres lo sigan manteniendo o de aquel hijo que por no ser aplicado en los estudios, es retirado de su institución educativa, y aun así, pretende seguir viviendo a costa de sus padres.

Sin embargo, consideramos que debemos ser muy cuidadosos al momento de evaluar si el hijo está abusando del ejercicio de su derecho a los alimentos, ya que se podría frustrar, injustificadamente, su derecho a la educación, como lo veremos más adelante.

Sobre lo que debemos entender por estudios exitosos, ya ha habido un pronunciamiento de la Corte Suprema en la Casación $N^{\circ}$ 1338-2004-LORETO comentada precedentemente:

Quinto.- ... solo en estos casos puede permitirse que un hijo mayor de edad pueda seguir percibiendo alimentos, siempre que curse dichos estudios de manera exitosa, los que deben entenderse realizados dentro de los márgenes razonables y aceptables, tanto en lo que se refiere al período de tiempo requerido para efectivizarlos, como a los resultados obtenidos, siendo esta la correcta interpretación de la norma acotada.

Nuevamente, concuerdo con la postura de la Corte Suprema, ya que cada caso concreto debe apreciarse de manera objetiva y razonable, para concluir, finalmente, si los estudios son exitosos o no.

Si bien en Argentina el obligado puede contradecir la acción acreditando que el hijo cuenta con recursos suficientes para proveerse los alimentos. 
Por otro lado, sobre este presupuesto "específico", Varsi (2011) ve la justificación de seguir brindando alimentos al hijo mayor de edad en el derecho a la educación y la capacitación para el trabajo. Por eso afirma que "este caso es lógico puesto que la obligación del padre incluye la educación superior que le ha de permitir al hijo ingresar al campo laboral y ejercer un trabajo digno" (p. 101).

Por su parte, Aguilar (2013) pone el énfasis en el estado de necesidad del hijo que se capacita:

Se justifica la norma en el entendido que seguir una carrera implica dedicación y tiempo, a la par de gastos propios de los estudios, por ello el alumno o alumna no tiene posibilidades de dedicarse a un trabajo que le reporte ingresos suficientes por estar atendiendo sus estudios. (p. 419)

Como podemos advertir, por lo menos, son tres las razones que sustentan este último presupuesto "específico" del derecho a los alimentos:

\section{i. La capacitación del hijo para el trabajo}

Conforme al artículo $472^{\circ}$ del CC, la capacitación para el trabajo forma parte de los alimentos, y ello tiene sentido, pues, si los alimentos tienen como fin primario mantener la subsistencia del alimentista, qué mejor forma de hacerlo que preparándolo para que pueda subsistir por sí mismo. Además de ello, el hijo que se capacita para el trabajo tiene mejores expectativas de obtener mayores ingresos que uno que no, por lo que siendo una de las características del derecho a los alimentos su reciprocidad (art. $474^{\circ}$ del $\mathrm{CC})$, aquel hijo podría en el futuro ser el obligado de dar los alimentos.

\section{ii. El estado de necesidad del hijo que se capacita}

Una parte del presupuesto "específico" bajo comento se apoya en el presupuesto "general" del estado de necesidad, lo que es entendible, si se toma en cuenta que seguir estudios de forma exitosa requiere de tiempo y esfuerzo, lo que provocaría que el hijo se encuentre imposibilitado de trabajar; sin embargo, sabemos que en nuestro medio, muchos jóvenes trabajan y estudian, a la vez, aunque lo tienen que hacer por necesidad y no por deseo. De todos modos, es evidente que el rendimiento en ambos quehaceres no será el mismo. En vista de ello, resulta razonable que se brinde alimentos al hijo o hija que está siguiendo estudios con éxito. 


\section{iii. La promoción del derecho a la educación}

Para entender más sobre este último punto, a continuación, explicaremos, brevemente, en qué consiste el derecho a la educación y por qué es importante promoverlo.

\section{EL DERECHO A LA EDUCACIÓN:}

El artículo $13^{\circ}$ de la Constitución Política del Perú establece que "la educación tiene como finalidad el desarrollo integral dela persona humana. ..."; y a continuación, el art. $14^{\circ}$ nos dice que "la educación promueve el conocimiento, el aprendizaje y la práctica de las humanidades, la ciencia, la técnica, las artes, la educación física y el deporte. Prepara para la vida y el trabajo y fomenta la solidaridad".

Así también, el artículo $2^{\circ}$ de la LGE expone sobre el concepto de educación lo siguiente:

La educación es un proceso de aprendizaje y enseñanza que se desarrolla a lo largo de toda la vida y que contribuye a la formación integral de las personas, al pleno desarrollo de sus potencialidades, a la creación de cultura, y al desarrollo de la familia y de la comunidad nacional, latinoamericana y mundial. Se desarrolla en instituciones educativas y en diferentes ámbitos de la sociedad.

Alvites (2010), por su parte, nos explica respecto del derecho a la educación que:

El derecho a la educación constituye un presupuesto indispensable para la democracia, así como para la plena realización y el ejercicio de otros derechos fundamentales ... como señala el Comité de Derechos Económicos, Sociales y Culturales, su satisfacción es un presupuesto para la superación de males que todavía subsisten en nuestra sociedad como la opresión contra la mujer, la pobreza o la explotación de los niños, niñas o adolescentes. Incluso, desde el punto de vista práctico, la inversión en educación que realiza un Estado constituye una de sus mejores inversiones de cara al desarrollo y crecimiento del país, tal como se expresa en el Acuerdo Nacional .... (p. 525)

Al igual que la capacitación para el trabajo, la educación también está comprendida dentro de los alimentos (art. $472^{\circ}$ del CC), lo que cobra mayor 
relevancia cuando se trata de menores de edad o de jóvenes en formación, ya que estos no podrían lograr un desarrollo integral sin educación ${ }^{54}$.

Dada la importancia y trascendencia de este derecho social, el cual también está previsto en instrumentos jurídicos supranacionales, como la Declaración Universal de Derechos Humanos, el Pacto Internacional de Derechos Económicos, Sociales y Culturales y el Pacto de San Salvador; el Estado, por razones de interés público, promueve la educación a través de diversas medidas como son los beneficios a determinados sectores. Por ejemplo: El pasaje universitario, la inafectación de impuestos para las instituciones educativas, el apoyo de un fondo (FONDEP) para el financiamiento de proyectos de las instituciones educativas, etc.

Con el mismo fin, el Estado puede imponer a los padres la obligación de garantizar los alimentos de sus hijos, más allá de su minoría de edad, siempre y cuando aquellos estén siguiendo con éxito estudios de una profesión u oficio ${ }^{55}$. Al respecto, resulta pertinente señalar que el artículo $54^{\circ}$ de la LGE establece lo siguiente:

La familia es el núcleo fundamental de la sociedad, responsable en primer lugar de la educación integral de los hijos. A los padres de familia, o a quienes hacen sus veces, les corresponde: a) Educar a sus hijos y proporcionarles en el hogar un trato respetuoso de sus derechos como personas, adecuado para el desarrollo de sus capacidades, y asegurarles la culminación de su educación.

\section{ELCRITERIOOBJETIVOCOMOPARÁMETROINTERPRETATIVO DE LOS ESTUDIOS EXITOSOS:}

Comentando el artículo $424^{\circ}$ del CC, Varsi (2011) nos dice que "la frase utilizada por el artículo, que estén siguiendo con éxito una profesión u oficio, es meramente subjetiva y dependerá del grado de apreciación de los padres y del juez." (p. 101).

No comparto su opinión, pues la manera particular de pensar y de sentir de cada uno no puede ser el parámetro para evaluar si los estudios de una profesión u oficio son exitosos, puesto que, de seguro, acabarán

54 En las STC de los Exps. N 4232-2004-AA/TC de fecha 19 de julio de 2006 y N N $^{\circ}$ 4646-2007-PA/TC de fecha 17 de octubre de 2007, el Tribunal Constitucional ha precisado que los fines constitucionales de la educación son: (i) promover el desarrollo integral de la persona; (ii) promover la preparación de la persona para la vida y el trabajo; y (iii) el desarrollo de la acción solidaria.

55 Cornejo (1999) el legislador puede, en nombre del interés colectivo, gobernar las relaciones familiares en el sentido que juzgue conveniente; pero únicamente en tanto no atropelle aquellos derechos ni traspase los límites señalados por la naturaleza a tales relaciones. ... (Cornejo Chávez, p. 20). 
en apreciaciones arbitrarias y sesgadas como las mencionadas en la parte introductoria de este artículo.

Tal vez la creencia de que el éxito es algo personal y meramente subjetivo, puede llevar a asumir que no puede haber objetividad en el criterio adoptado. Nosotros demostraremos que eso no es así, y para ello, recurriremos, primero, al significado del término.

Según la Real Academia Española (RAE, 2019), el vocablo "éxito" significa: "1. m. Resultado feliz de un negocio, actuación, etc. 2. m. Buena aceptación que tiene alguien o algo. 3. m. p. us. Fin o terminación de un negocio o asunto."

Como podemos observar, la palabra "éxito" tiene tres acepciones: La primera de ellas hace referencia a resultados satisfactorios ${ }^{56}$; la segunda, a una consideración positiva (la buena aceptación); y la tercera acepción, aunque menos usada, hace alusión, simplemente, a la culminación de algún emprendimiento.

De lo expuesto, tenemos que, de las tres acepciones del término, solo la segunda importa subjetividad; mientras que la primera y la tercera acepción están relacionadas al logro de objetivos o metas, aspectos que pueden estar basados en datos y ser perceptibles por los sentidos.

Considerar el éxito en los estudios como la buena aceptación que se tiene de estos o del estudiante, conllevará muchas veces a respuestas disímiles, lo que restaría predictibilidad al asunto; por el contrario, adoptar un criterio objetivo y considerar el éxito de dichos estudios como el logro o la culminación de objetivos y metas académicas por parte del estudiante, es algo que se puede valorar sin mayores apasionamientos, y por tanto, sería un criterio adecuado, máxime, si vemos que dos de los tres sentidos de la palabra "éxito" lo conciben así.

Esta concepción también es compatible con los fundamentos del derecho a los alimentos que hemos analizado y la promoción del derecho a la educación, ya que, si se opta por un criterio subjetivo, es muy probable que la exigencia al hijo sea mayor (solemos apreciar el éxito como una posición a la que pocas personas llegan: p. ej., exigirle al estudiante una nota promedio superior a 15 para estimar que sus estudios son exitosos).

Nótese la semejanza con el artículo $109^{\circ}$ del CFPF boliviano cuando señala: "siempre y cuando la dedicación a su formación evidencie resultados efectivos". 
En ese sentido, para poder determinar si el hijo o hija, solteros y mayores de edad, están siguiendo con éxito estudios de una profesión u oficio (lo que significa que tendrían derecho a recibir alimentos de sus padres), proponemos que en cada caso concreto, se evalúe si están logrando sus objetivos académicos y resulta factible que inicien o culminen sus estudios hasta su habilitación; valorando en todo momento datos objetivos como la edad de los hijos, la carrera profesional u oficio emprendido, la exigencia de la institución educativa, sus notas académicas, entre otros.

Esto explica, por qué se puede considerar como exitosos los estudios de un alumno con un promedio ponderado igual o mayor de 11, siempre y cuando, tenga la posibilidad académica de proseguir y culminar sus estudios ${ }^{57}$.

\section{CONCLUSIONES}

- El concepto de alimentos ha ido evolucionando, por lo que ya no solo comprende lo necesario para la subsistencia material del alimentista; sino también, y con la misma importancia, sus necesidades mentales y emocionales, de tal forma que está orientado al desarrollo integral del alimentista. En la actualidad, incorpora aspectos como la capacitación para el trabajo, la asistencia psicológica y la recreación del alimentista sin distingos de edad.

- Los CC de Argentina, Colombia y Chile contienen normas para presumir del estado de necesidad de los hijos mayores de edad y menores de 21 años. Esto significa que, en principio, tienen derecho a recibir alimentos de sus padres hasta alcanzada aquella edad. Argentina, Chile y Bolivia, cuentan con disposiciones para beneficiar con alimentos a los hijos mayores de edad que cursan estudios de una profesión u oficio; sin embargo, Bolivia es el único país que exige como condición, que dichos estudios produzcan "resultados efectivos", siendo, por tanto, su regulación, la más parecida a la nuestra.

- La doctrina acepta pacíficamente que los presupuestos "generales" del derecho a los alimentos son 3: a) El estado de necesidad del alimentista; b) Las posibilidades del alimentante; y c) La norma legal que establezca la obligación.

Nótese la semejanza con el artículo $109^{\circ}$ del CFPF boliviano cuando señala: "siempre y cuando la dedicación a su formación evidencie resultados efectivos". 
- El estado de necesidad es una situación de hecho en la que el individuo no puede de proveerse los alimentos por sí mismo, ya sea por razones intrínsecas o extrínsecas. La doctrina admite que cuestiones como la inmadurez y el desempleo, que bien sabemos, caracterizan la vida de muchos de nuestros jóvenes, pueden ser la causa del estado de necesidad.

- Son 2 los supuestos de hecho regulados por el art. $424^{\circ}$ del CC en los que corresponde a los hijos (e hijas) que han adquirido la mayoría de edad, seguir recibiendo alimentos de sus padres: a) Cuando sean solteros (o solteras) y estén siguiendo con éxito estudios de una profesión u oficio hasta la edad de 28 años; y b) Cuando sean solteros (o solteras) y no se encuentren en aptitud de atender a su subsistencia por causas de incapacidad física o mental debidamente comprobadas.

- Los presupuestos "específicos" del derecho a los alimentos en el primer supuesto de hecho del artículo $424^{\circ}$ del CC serían 4: a) El vínculo filial; b) El estado civil de soltero o soltera; c) El rango de edad entre los 18 y los 28 años; d) Los estudios exitosos de una profesión u oficio.

- En el ámbito jurisprudencial, se ha establecido que los estudios exitosos también comprenden los estudios preparatorios o previos, requeridos para el acceso a los estudios superiores, vale decir, la educación básica y los estudios de preparación para el ingreso a universidades, institutos y escuelas de educación superior.

- La jurisprudencia también ha resuelto que debemos entender por estudios exitosos aquellos que han sido realizados dentro de los márgenes razonables y aceptables, tanto en lo que se refiere al período de tiempo requerido para efectivizarlos, como a los resultados obtenidos.

- La razón por la que la norma del art. $424^{\circ}$ del CC exige que los estudios sean exitosos, sería la prohibición del ejercicio abusivo del derecho previsto en el art. II del Título Preliminar del CC, toda vez que tampoco se quiere fomentar el ocio o el engaño.

- Consideramos que, por lo menos, son 3 las razones que sustentan el derecho a los alimentos para estos hijos solteros (o solteras) mayores de edad que están siguiendo con éxito una profesión u 
oficio: a) La capacitación del hijo para el trabajo; b) El estado de necesidad del hijo que se capacita; y c) La promoción del derecho a la educación.

- El derecho a la educación es un derecho fundamental recogido en la Constitución y normas supranacionales. Hemos podido apreciar que este derecho trasciende su esencia y permite realizar otros derechos fundamentales, siendo valorado, incluso, como motor de desarrollo del país. De allí que resulta de interés público promover la educación a través de diversas medidas. Como una forma de promover la educación, el Estado puede imponer a los padres la obligación de prestar alimentos a sus hijos, no obstante haber alcanzado la mayoría de edad, siempre y cuando, se presenten algunos presupuestos como los analizados en este artículo.

- Proponemos que se debe valorar el éxito del hijo (o hija) que se capacita para el trabajo con un criterio objetivo, partiendo de 2 de las acepciones del término en cuestión, lo que brindaría una mayor predictibilidad y sería compatible con los fundamentos del derecho a los alimentos y la promoción del derecho a la educación. Por lo tanto, recomendamos que en cada caso concreto, se debe evaluar si el estudiante está logrando sus objetivos académicos y resulta factible que inicie o culmine sus estudios hasta su habilitación; valorando en todo momento datos objetivos.

\section{REFERENCIAS}

Aguilar, B (2013). Derecho de Familia. Lima: Ediciones Legales E.I.R.L.

Alvites, E. (2010). Perspectivas constitucionales sobre el derecho fundamental a la educación. Los derechos fundamentales. Estudios de los derechos constitucionales desde las diversas especialidades del Derecho. Lima: Gaceta Jurídica S.A.

Bossert, G. y Zanonni, E. (2016). Manual de Derecho de Familia. $7^{a}$ edición actualizada y ampliada. Buenos Aires, Argentina: Editorial Astrea.

Cabanellas, G. (1979). Diccionario enciclopédico de Derecho usual. Tomo 1, $12^{\mathrm{a}}$ edición. Buenos Aires, Argentina: Editorial Heliasta S.R.L.

Cornejo, H. (1999). Derecho familiar peruano. Sociedad conyugal, sociedad paterno filial, amparo familiar del incapaz. 10ª edición. Lima: Gaceta Jurídica S.A. 
Chunga (2011). Código Civil comentado. Tomo 3, Derecho de Familia (segunda parte), $3^{a}$ edición. Lima: Gaceta Jurídica S.A.

Díez-Picazo y Gullón (1998). Sistema de Derecho Civil. Vol. 4, 7ª edición. Madrid, España: Editorial Tecnos S.A.

Hernández, C. (2011). Código Civil comentado. Tomo 3, Derecho de Familia (segunda parte), $3^{\mathrm{a}}$ edición. Lima: Gaceta Jurídica S.A.

Jiménez, F. (2012). Una "revisitación", ante las situaciones de necesidad de los niños, de una institución ya centenaria: La obligación de alimentos. Observatorio de Derecho Civil. Vol. 12, 2ª edición. Lima: Motivensa S.RL.

Real Academia Española (2014). Diccionario de la lengua española [versión electrónica]. 23 $3^{a}$ edición. Madrid, España: Espasa, https://dle.rae. es $/ \% C 3 \%$ A 9 xito? $m=$ form

Varsi, E. (2011). Código Civil comentado. Tomo 3, Derecho de Familia (segunda parte), $3^{\underline{a}}$ edición. Lima: Gaceta Jurídica S.A.

Varsi, E. (2012). Tratado de Derecho de Familia. Derecho familiar patrimonial. Relaciones económicas e instituciones supletorias y de amparo familiar. Tomo 3. Lima: Gaceta Jurídica S.A. 